\title{
Neue Wachstumskurven: für Schweizer Kinder nicht repräsentativ
}

\author{
Die alten Wachstumskurven basierten auf Daten Zürcher Kinder, die neuen Kurven \\ hingegen zu einem grossen Teil auf Daten amerikanischer Kinder. Diese sind infolge \\ ethnischer Unterschiede kleiner und ihre Pubertät beginnt früher. Die Autoren be- \\ klagen, dass die neuen Wachstumskurven vor allem im Bereich des späteren Kindes- \\ und Pubertätsalters eine Verschlechterung darstellen.
}

Urs Eiholzer, Udo Meinhardt

Die Langfassung dieses Beitrags und ein Diskussionsforum finden sich auf www.pezz.ch

Eine Stellungnahme findet sich im nächsten Beitrag.

Vor kurzem haben das Zürcher Kinderspital und die Schweizer Gesellschaft für Pädiatrie neue Wachstumskurven publiziert. Die bisherigen Wachstumskurven stammten von Zürcher Kindern, während die neuen auf verschiedensten in- und ausländischen, vor allem amerikanischen Daten beruhen. Für die leichte Verbesserung dieser neuen Kurven für das erste Lebensjahr wird eine gravierende Verschlechterung im späteren Kindes- und Pubertätsalters in Kauf genommen. Die amerikanischen Kinder, auf deren Daten die neuen Kurven basieren, sind infolge von ethnischen Unterschieden kleiner. Auch findet der Pubertätswachstumsspurt mehr als zwei Jahre früher statt als bei den Schweizer Kindern.

\begin{tabular}{ll}
\hline $\begin{array}{l}\text { Bisherige Kurven } \\
\text { 0-18 Jahre }\end{array}$ & $\begin{array}{l}\text { Grösse, Gewicht, BMI, Kopfumfang, Sitzhöhe (Körperproportionen) } \\
\text { Wachstumsgeschwindigkeit, Hautfalten usw.: 1. Zürcher Longitu- } \\
\text { dinalstudie (1. ZLS), 1955-1975 }\end{array}$ \\
Neue Kurven & $\begin{array}{l}\text { Grösse, Gewicht, BMI. WHO-Daten aus Brasilien, Ghana, Indien, } \\
\text { Norwegen, Oman und USA }\end{array}$ \\
\hline 5-18 Jahre & $\begin{array}{l}\text { Grösse, Gewicht, BMI. Bis 10 Jahre unbearbeitete, danach bearbei- } \\
\text { tete US-Daten von 1977 (Weisse, Schwarze, Hispanos, Asiaten) }\end{array}$ \\
\hline 0-18 Jahre & $\begin{array}{l}\text { Kopfumfang. Neu berechnete Daten aus der 2. Zürcher Longitu- } \\
\text { dinalstudie (2. ZLS) (1975-1995) }\end{array}$ \\
\hline 1-18 Jahre & $\begin{array}{l}\text { Wachstumsgeschwindigkeit, Körperproportionen. Unbearbeitete } \\
\text { Kurven der 1. ZLS (s. o.). }\end{array}$ \\
\hline
\end{tabular}

Korrespondenz:

Prof. Dr. med. Urs Eiholzer Pädiatrisch-Endokrinologisches Zentrum Zürich PEZZ

Möhrlistrasse 69

CH-8006 Zürich

urs.eiholzer@pezz.ch

\section{Weshalb die bisherigen Kurven auch heute noch besser sind}

\section{Erstes Lebensjahr}

Die neuen roten Säuglingskurven sind denen der bisherigen schwarzen Kurven sehr ähnlich. Die maximale Differenz der 50. Längenperzentile beträgt im Alter von drei Monaten knapp $1 \mathrm{~cm}$. Beim Gewicht zeigt sich ein rascherer Anstieg. Die neusten Schweizer Daten der ZAPP-Studie (Zürcher Arbeitsgemeinschaft praktizierender Pädiater, Eiholzer 1998) zeigen aber, dass Schweizer Kinder mit einem Jahr noch schwerer und grösser sind.

\section{Grösse 1-18 Jahre}

Die Unterschiede zwischen den neuen und den bisherigen Wachstumskurven beginnen im Alter von ein bis zwei Jahren und bleiben bis zum Pubertätswachstumsspurt bestehen. Mädchen und Frauen sind auf den neuen Wachstumskurven $2 \mathrm{~cm}$ kleiner als auf den bisherigen. Dieser Unterschied verschwindet wegen der früheren Pubertät der Amerikanerinnen vorübergehend. Knaben sind auf den neuen Kurven $4 \mathrm{~cm}$ kleiner als auf den bisherigen. Auch dieser Unterschied verschwindet mit 10 Jahren wegen der früheren Pubertät der Amerikaner.

\section{Gewicht 1-18 Jahre}

Die oberen Perzentilen der neuen Kurven liegen deutlich über den bisherigen - wahrscheinlich zu hoch. Hinter den Normwerten für Gewicht und Grösse liegen unterschiedliche Problemstellungen. Die Normwerte der amerikanischen Realität anzupassen, führt zu einem problematischen permissiven Effekt.

\section{Die neuen Kurven sind wenig repräsentativ}

Die neuen Wachstumskurven sind wegen ethnischer Unterschiede für die Schweiz wenig repräsentativ.

- Das Grössenwachstum einer Gesellschaft wird mit zunehmendem Wohlstand bis zu einem genetisch vorgegebenen Maximum optimiert. Dieser sekuläre Trend kann gut an den Daten der Rekrutenaushebungen verfolgt werden. Aufgrund der neuen Wachstumskurven wären die heutigen Schweizer Knaben und Mädchen/Frauen kleiner als vor 50 Jahren. Die Logik des sekulären Trends spricht aber für eine Zunahme der Grösse: So sind die Männer (Aushebung 2004) im Durchschnitt 
Abbildung 1

Kurvenvergleich (Beispiele). Schwarz: alte Wachstumskurven; rot: neue Wachstumskurven; Jeweils 3., 50. und 97. Perzentile. Grün auf Säuglingskurve: ZAPP-Kurve, 50. Perzentile (Zürcher Arbeitsgemeinschaft praktizierender Pädiater).

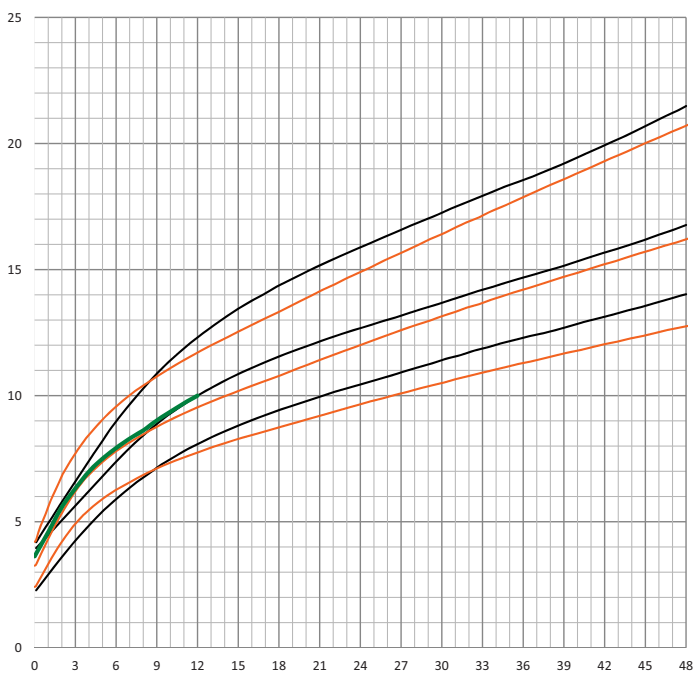

Gewicht Knaben 0-48 Monate

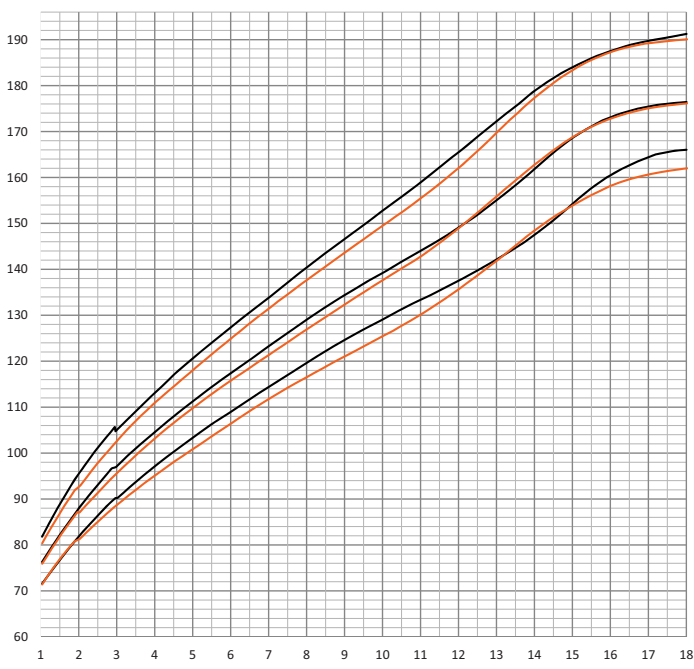

Grösse Knaben 1-18 Jahre

$177,5 \mathrm{~cm}$ gross, also grösser als nach den bisherigen und den neuen Wachstumskurven (50. Perzentile: $176,0 \mathrm{~cm})$. Weil die Daten der 1. Zürcher Longitudinalstudie (1. ZLS) aus der Mittelschicht stammen und sich in der Zwischenzeit der Wohlstand auf breite Kreise verteilt hat, sind diese Daten noch heute repräsentativ.

- Für grössere Schweizer Kinder spricht auch eine neue Arbeit, die zeigt, dass der Kopfumfang sehr gut mit der Körpergrösse korreliert. Weil der Kopfumfang von Schweizer gegenüber US-Kindern grösser ist, wurden für die neuen Kurven die Daten der 2. ZLS verwendet.

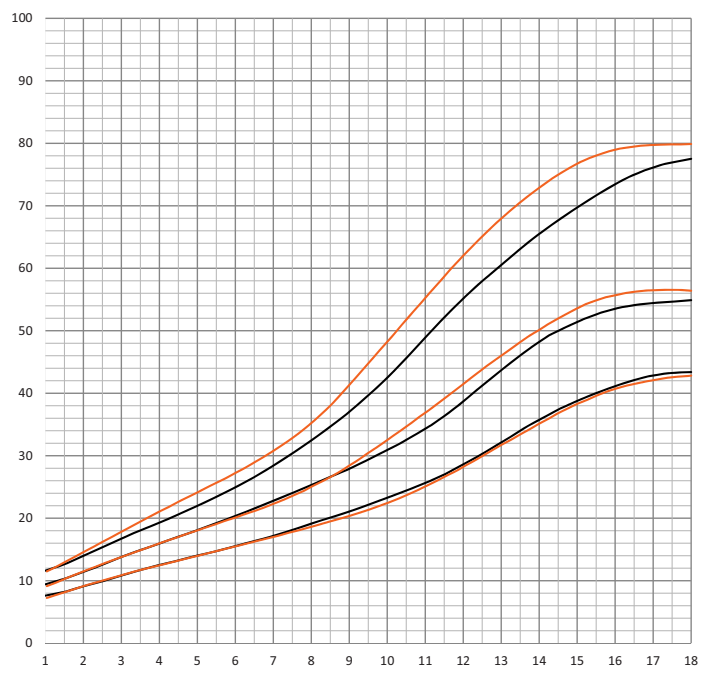

Gewicht Mädchen 1-18 Jahre

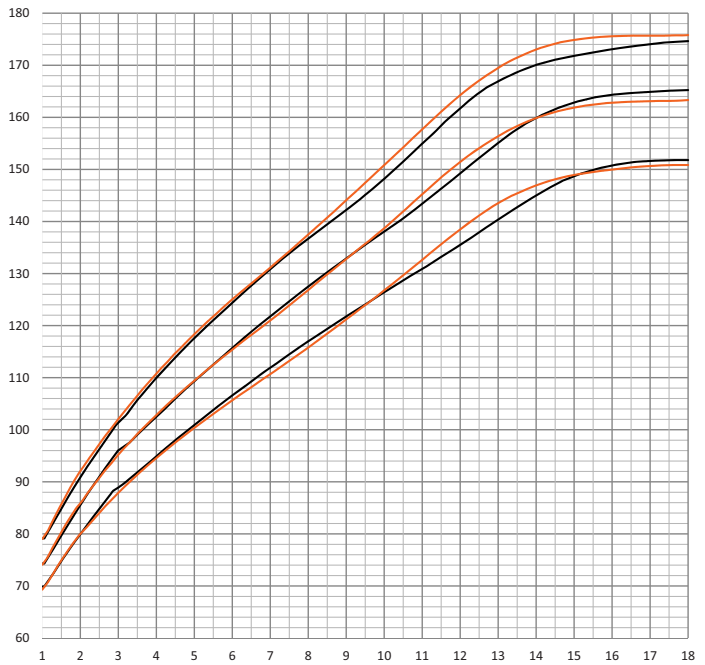

Grösse Mädchen 1-18 Jahre

- Gerade wegen des Timings des Pubertätswachstumsspurtes, der auf den neuen Kurven rund 2 Jahre zu früh erfolgt, fordern Wachstumsspezialisten, dass bevölkerungsspezifische Daten verwendet werden.

\section{Wie weiter?}

Kurzfristig sollten wir die bisherigen Wachstumskurven weiterverwenden. Mittelfristig sollten sie mit punktuellen Kontrollen angepasst werden. Zusätzlich sollten die Daten der 2. ZLS sowie der Generationenstudie verwendet werden, für deren Auswertung der Nationalfonds 3,9 Millionen Franken zur Verfügung gestellt hat. 